\title{
10 SUPORTE EMOCIONAL ÀS GESTANTES QUE CONVIVEM COM DOENÇAS CRÔNICAS ${ }^{1}$
}

| Pâmela da Silveira²; Claudia Tavares ${ }^{3}$; Fernanda Marcondes ${ }^{4} \mid$

\section{RESUMO}

CONTEXTO: A mulher que convive com a doença crônica se tornará uma grávida de alto risco e poderá encontrar dificuldades para as adaptações emocionais impostas pela nova realidade.

OBJETIVO: Discutir a demanda de acolhimento emocional das gestantes que convivem com doença crônica e o trabalho dos enfermeiros no pré-natal de alto risco.

METODOLOGIA: Pesquisa qualitativa de abordagem sociopoética, realizada no Ambulatório de Pré-Natal do Hospital Universitário Antônio Pedro, Niterói/RJ. Participaram do estudo, 06 gestantes em acompanhamento pré-natal que convivem com doenças crônicas. A produção de dados se deu por meio de técnica criativa através da "linha da vida gestacional" e do dispositivo grupo-pesquisador. A análise de dados utilizada foi a análise de conteúdo temático-categorial.

RESULTADOS: Em decorrência da formação - o foco da atenção do enfermeiro se mantém ligado à doença ou as suas formas de expressão - sinais e sintomas, o que torna o estado emocional da mulher, assim como, os fatores sociais esquecidos no seguimento de sua gestação.

CONCLUSÃO: Há necessidade de escuta ativa aos anseios em todo o seguimento da gestação de alto risco, pois as mulheres se encontram fragilizadas e instáveis, e não encontram espaço para exporem seus medos, angústias e expectativas, acolhimento emocional que deve ser realizado por enfermeiros

PALAVRAS-CHAVE: Gestante; Doenças crônicas; Saúde mental; Pré-natal

\section{RESUMEN}

"El apoyo emocional a las mujeres embarazadas con enfermedades crónicas"

CONTEXTO: La mujer que vive con la enfermedad crónica se convertirá en una embarazada de alto riesgo y puede encontrarse con dificultades en los ajustes emocionales impuestos por la nueva realidad. OBJETIVO: Analizar la demanda de apoyo emocional de las mujeres embarazadas que viven con una enfermedad crónica y el trabajo de las enfermeras en la atención prenatal de alto riesgo.

METODOLOGÍA: La investigación cualitativa del enfoque poética llevada a cabo en la clínica prenatal del Hospital de la Universidad Antonio Pedro, Niterói / RJ. 06 mujeres embarazadas que viven con enfermedades crónicas participaron en control prenatal. Los datos se produjeron a través de la técnica creativa por "línea de vida gestacional" y el dispositivo grupo-investigador. El análisis de datos fue el medio de análisis del contenido de temática categórica.

RESULTADOS: Como resultado de la formación - el foco de atención de la enfermera sigue siendo la enfermedad o sus formas de expresión - signos y síntomas, lo que hace que el estado emocional de la mujer, así como los factores sociales sean olvidados tras su embarazo.

CONCLUSIÓN: Existe la necesidad de una escucha activa de las preocupaciones a lo largo de los embarazos de alto riesgo, porque las mujeres se encuentran frágiles e inestables, y no hallan un lugar para exponer sus miedos, ansiedades y expectativas, cuidado emocional que debe ser realizada por personal de enfermería.

\section{DESCRIPTORES: Embarazada; Enfermedades crónicas; Salud mental; Prenatal}

\begin{abstract}
"Emotional support to pregnant women living with chronic diseases"

BACKGROUND: Women who live with chronic disease will become high-risk pregnant and may encounter difficulties in the emotional adjustments imposed by the new reality.

AIM: To discuss the demand of emotional assistance of pregnant women living with chronic illness and the work of nurses in high-risk prenatal care.

METHODS: Qualitative research of the social poetic approach held in Prenatal Clinic of Antonio Pedro University Hospital, Niterói / RJ. 06 pregnant women in prenatal care living with chronic diseases participated in the study,. Data was collected through creative technique by "line of gestational life" and group-researcher device. Data analysis was used to analyze categorical-themed content.

RESULTS: As a result of training - the focus of the nurse's attention remains attached to the disease or its forms of expression - signs and symptoms, which produces the emotional state of women, as well as forgotten social factors following her pregnancy.

CONCLUSION: There is the need of active listening the concerns throughout the follow-up of high-risk pregnancies due to women's fragility and instability, and the lack of room to expose their fears, anxieties and expectations, emotional care that should be performed by nurses.
\end{abstract}

\section{KEYWORDS: Pregnant; Chronic diseases; Mental health; Pre- natal}

Submetido em 22-02-2016

Aceite em 30-05-2016

1 Este artigo é parte do trabalho de conclusão de curso intitulado "Estratégias de promoção à saúde mental com gestantes que convivem com doenças crônicas", apresentado no I Encontro Internacional Inovação no Ensino na Saúde e Enfermagem.

2 Enfermeira; Membro do Núcleo de Pesquisa: Ensino, Criatividade e Cuidado em Saúde e Enfermagem; Mestranda do Programa de Mestrado Acadêmico em Ciências do Cuidado em Saúde pela Universidade Federal Fluminense, Rua Dr. Celestino, 74, 24020-091 Niterói/RJ, Brasil, pamelagioza@hotmail.com

3 Enfermeira; Doutora em Enfermagem pela Universidade Federal do Rio de Janeiro;; Coordenadora do Programa de Pós-Graduação em Enfermagem e do Curso de Mestrado Profissional Ensino na Saúde; Professora Titular na Universidade Federal Fluminense, Escola de Enfermagem Aurora de Afonso Costa, Brasil, claudiamarauff@gmail.com 4 Enfermeira; Membro do Núcleo de Pesquisa: Ensino, Criatividade e Cuidado em Saúde e Enfermagem; Mestre em Ciências do Cuidado em Saúde pela Universidade Federal Fluminense, 24020-091 Niterói/RJ, Brasil, fe_laxe@yahoo.com.br

Citação: Silveira, P., Tavares, C., \& Marcondes, F. (2016). Suporte emocional às gestantes que convivem com doenças crônicas: demandas de trabalho emocional do enfermeiro. Revista Portuguesa de Enfermagem de Saúde Mental (Spe. 4), 63-68. 


\section{INTRODUÇÃO}

A gestação é uma experiência única na vida de uma mulher. Nesse período, ocorrem mudanças físicas, sociais e emocionais que são capazes de gerar ansiedade, medo, novas descobertas e expectativas, o que requer instruções e ensinamentos para auxiliá-la no que tange a gestação, autocuidado, preparo para o parto e para a maternidade (Vasques, 2006). Por ser um fenômeno fisiológico, a gestação, tem o seu desenvolvimento, na maioria dos casos, sem intercorrências. Entretanto, algumas mulheres por apresentarem algum tipo de patologia crônica, sofrerem algum dano ou desenvolverem problemas, se tornam vulneráveis a evoluir para uma gestação prejudicial, tanto para a mãe quanto para o feto (Ministério da Saúde, 2012).

A doença crônica, por muitas vezes, pode significar uma ameaça aos planos futuros de vida dos indivíduos acometidos pela mesma, pois lhes são impostas grandes mudanças nos seus padrões de consumo, hábitos e rotina, além do controle permanente de sinais e sintomas que, se não mantidos dentro dos padrões, podem progredir para níveis mais graves e ocasionar a morte (Quevedo, Lopes, e Lefevre, 2006). A mulher que convive com a doença crônica se tornará uma grávida de alto risco. Em virtude disso, poderá encontrar dificuldades para as adaptações emocionais impostas pela nova realidade. Em consequência da patologia se tornar um fator de risco à gestação, acabam surgindo determinadas situações tais como: o medo em relação a sua própria saúde e a do bebê; as modificações que estão ocorrendo com seu corpo; o receio de seu filho nascer com alguma anormalidade e, adicionado a esses fatores, a perda de controle em relação à gravidez e a si mesma (Quevedo, 2010). Urge que o enfermeiro no pré-natal desenvolva conhecimentos e sensibilidade para detectar e entender o processo emocional que rodeia o acompanhamento da gestação de alto risco (Ministério da Saúde, 2012).

A assistência ao pré-natal tem conquistado cuidado especial na saúde materno-infantil, em decorrência de taxas desfavoráveis relacionada aos coeficientes de mortalidade materna e infantil o que tem estimulado o desenvolvimento de políticas voltadas ao ciclo gestacional e puerperal, como o Programa de Humanização no Prénatal e Nascimento (PHPN) (Duarte, 2012).

Tal programa visa assegurar a melhoria do acesso, da cobertura e da assistência ao parto e puerpério às gestantes e aos recém-nascidos. Entre as ações realizadas pelo programa estão: exames laboratoriais básicos e os procedimentos obstétricos considerados essenciais, classificados como adequados, inadequados ou intermediários (Duarte, 2012).
Dessa forma, o pré-natal apresenta-se como um conjunto de ações voltadas à mulher grávida e ao bebê, que visa: diagnosticar ou confirmar enfermidades maternas; realizar o tratamento; acompanhar a evolução da gravidez a partir das condições da gestante e o desenvolvimento fetal; tratar as complicações clínicas referentes à gravidez; orientar quanto medidas preventivas para saúde da gestante/feto e instruir a mãe para o momento do parto e o posterior aleitamento (Caldas, Silva, Boing, Crepaldi, e Custódio, 2013).

Com a ampliação do Programa Saúde da Família, a assistência a gestante no pré-natal ocorre de forma multidisciplinar, permitindo que as condutas e cuidados sejam compartilhados (Duarte, 2012).

De acordo com o funcionamento atual do Sistema Único de Saúde, a atenção básica é responsável por realizar atendimento ambulatorial de pré-natal de gestantes que não apresentam risco e por encaminhar as gestantes identificadas como "alto risco" para os serviços de referência (Caldas et al., 2013)

O objetivo da assistência pré-natal de alto risco consiste em intervir para diminuir os danos que as complicações relacionadas à patologia materna possam vir a causar para a mãe e para o feto. Com isso, o enfermeiro como integrante da equipe multiprofissional, deve estar preparado para enfrentar quaisquer fatores que possam afetar a gravidez, em uma visão integral, ou seja, considerando os aspectos clínicos, socioeconômicos e emocionais. (Ministério da Saúde, 2012)

Entretanto diante da complexidade envolvida em gestações de mulheres que convivem com doenças crônicas (gestação de alto risco), a assistência de saúde prestada se limita a consulta médica individual, que tem como foco a patologia e como esta influencia na vida da mãe e do feto, não sendo abordadas questões que envolvam o estado emocional da gestante e como a mesma enfrenta as situações impostas pela doença.

Maçola, Vale e Carmona (2010) relatam que estudos evidenciam a importância da atenção à saúde mental da gestante e sua relação com o desenvolvimento do vínculo mãe-filho. Porém, ainda é escassa a realização de programas de pré-natal que abordem efetivamente as questões emocionais, visando auxiliar a mulher a lidar com o processo de gestação e maternidade.

Dessa forma, a inserção de estratégias de promoção da saúde mental das gestantes no âmbito do pré-natal se torna essencial, pois visa minimizar os efeitos negativos que a presença da patologia na gravidez possa gerar. Para Guerra, Braga, Quelhas e Silva (2014) algumas estratégias definidas como promotoras de saúde mental em gestantes são: identificação precoce de fatores de risco, estabelecimento de uma relação de confiança com o 
enfermeiro especialista em saúde materna e obstétrica, preparação para o desempenho do papel, identificação da rede de suporte social, incentivar o envolvimento do pai na vigilância da gravidez nos cuidados com a criança e realização de cursos de preparação para a parentalidade em grupo.

De acordo com Arrais, Mourão e Fragalle (2014) quando a assistência pré-natal abrange, além do aspecto biológico, continente ou base consistente para a mulher e sua família, o enfermeiro torna o cuidado obstétrico humanizado e integral, o que contribui para a redução da morbimortalidade materna e transforma a realidade vivenciada pelas mulheres durante o período da gravidez. Para Shimizu e Lima (2009) a consulta de enfermagem contribui para que a gestante enfrente esta etapa da vida com mais tranquilidade, pois lhe permite compreender e expressar os diversos sentimentos vivenciados. Além disso, o enfermeiro é o profissional habilitado e de fácil acesso as mulheres no acompanhamento gestacional, devendo acolher não só a gestante, mas ao contexto familiar e social em que a mesma está inserida, prestando uma assistência integral e qualificada, proporcionando assim uma gravidez tranquila e feliz à mulher e o nascimento de um bebê saudável (Santos, 2014).

O enfermeiro deve acolher a gestante e sua família de forma a ouvir suas dúvidas e questionamentos, sem julgamentos ou preconceitos, de forma a criar um vínculo profissional de confiança. É fato que a assistência à mulher durante o pré-natal já não se satisfaz mais com medidas biomédicas, a gestação passou a ser vista como um momento de mudanças não apenas fisiológicas, mas também, como um momento de profundas transformações emocionais e biopsicossociais, onde cada casal a vivencia de forma única, exigindo, portanto, atenção individualizada e holística (Santos, 2014). Este contexto de acolhimento e humanização reforça o profissional enfermeiro como elemento ativo da equipe de saúde, por exercer papel educativo e contribuir para que se produzam mudanças concretas e saudáveis, nas atividades das gestantes, familiares e comunidade, buscando o bem-estar e qualidade de vida (Lima e Moura, 2005). Assim, o trabalho do enfermeiro com as emoções das mulheres que convivem com doenças crônicas no âmbito do pré-natal contribui com a promoção da sua saúde mental, favorecendo a exposição de sentimentos, como expectativas perante a maternidade e aflições decorrentes do convívio com a doença crônica e outros problemas relacionados direta ou indiretamente à gravidez. Contudo, ainda são escassos os estudos que abordam as potencialidades do enfermeiro no âmbito do pré-natal, assim como, no período investigado foram inexistentes pesquisas que referissem o trabalho do enfermeiro no acolhimento emocional prestado as gestantes, numa abordagem humanizada e holística destinada a estas mulheres.

\section{METODOLOGIA}

Trata-se de um estudo qualitativo, de abordagem sociopoética, realizado no ambulatório de Pré Natal de alto risco do Hospital Universitário Antônio Pedro (HUAP), Niterói/RJ. As participantes da pesquisa foram definidas a partir dos seguintes critérios de inclusão: gestantes que convivem com doenças crônicas como Hipertensão Arterial Crônica e Diabetes Mellitus, patologias mais frequentes no ambulatório de pré-natal. Como critério de exclusão as gestantes menores de 18 anos, as que estavam acompanhadas de crianças e as que apresentaram algum tipo de intercorrência clínica no momento da coleta de dados. Aplicando-se os critérios estabelecidos participaram do grupo-pesquisador 6 gestantes.

A produção de dados baseada nos princípios da sociopoética se deu por meio da constituição do grupopesquisador. A sociopoética é um método de pesquisa que visa à produção de subjetividade, utilizando a sensibilidade, a criatividade e a relação com o outro. Essa abordagem de pesquisa se concretiza através dos seguintes pontos: a importância do corpo como fonte de conhecimento; a importância das culturas dominadas e de resistência; das categorias e dos conceitos que elas produzem; papel dos sujeitos pesquisadores como co-responsáveis pelos conhecimentos produzidos, "copesquisadores", papel da criatividade de tipo artístico no aprender, no conhecer e no pesquisar; a importância do sentido espiritual, humano, das formas e dos conteúdos no processo de construção dos saberes (Moraes, Braga, e Silveira, 2003).

Para produção criativa dos dados desenvolveu-se a técnica da "linha da vida gestacional" na qual as gestantes dividiriam um papel cartolina em três etapas $\left(1^{\circ}, 2^{\circ} \mathrm{e}\right.$ $3^{\circ}$ trimestre gestacional) e ali através de desenhos ou de figuras de revistas ilustrariam os principais acontecimentos que marcaram sua gestação, seguido da questão que norteou o grupo- pesquisador: Você se sente acolhida emocionalmente pela equipe de saúde durante a realização do pré-natal? Recebe algum tipo de apoio emocional por parte dos profissionais durante as consultas de pré-natal realizadas no ambulatório?

A inspiração da linha da vida gestacional se deu a partir da técnica linha da vida, que segundo Szenészl (2011) é uma representação da organização espacial do tempo de uma pessoa. Tem a particularidade de indicar características do modo como ela se organiza e se adapta em sua relação com seu meio externo, sendo também uma 
metáfora sobre como ela vê, sente e interpreta aspectos de sua vida emocional. Essa técnica é a via de acesso à representação dos eventos significativos da vida do cliente. Os dados produzidos com as gestantes foram submetidos a técnica de análise de conteúdo temático-categorial. As etapas definidas por Bardin (2009) para tal análise são: pré-análise; exploração do material ou codificação; tratamento dos resultados, inferência e interpretação. Essas etapas podem ser especificadas como: Primeira Etapa (Pré-análise): Nesta etapa são desenvolvidas as operações preparatórias para a análise propriamente dita. Consiste num processo de escolha dos documentos ou definição do corpus de análise; formulação das hipóteses e dos objetivos da análise; elaboração dos indicadores que fundamentam a interpretação final. Segunda Etapa (Exploração do material ou codificação): Consiste no processo através do qual os dados brutos são transformados sistematicamente e agregados em unidades, as quais permitem uma descrição exata das características pertinentes ao conteúdo expresso no texto. Terceira Etapa (Tratamento dos resultados - inferência e interpretação): Busca-se colocar em relevo as informações fornecidas pela análise, através de quantificação simples (frequência) ou mais complexas como a análise fatorial, permitindo apresentar os dados em diagramas, figuras, modelos etc. Respeitando os preceitos da resolução 466/2012, do Conselho Nacional de Saúde/ Ministério da Saúde, o trabalho foi submetido ao Comitê de Ética em Pesquisa da Faculdade de Medicina da Universidade Federal Fluminense (UFF), cumprindo o que preceitua a Resolução no 466/12 (CNS), e aprovado segundo $n^{\circ}: 45480915.5 .0000 .5243$.

\section{RESULTADOS}

Após a análise dos dados obtidos, os resultados estão dispostos abaixo, através do Perfil sócio-demográfico e Gestacional das mulheres e da seguinte categoria de análise "Alterações emocionais vivenciadas a cada trimestre gestacional", na qual está inserido o seguinte tema "Demandas de acolhimento emocional".

\section{Perfil Sócio-Demográfico e Gestacional}

A maioria das gestantes participantes do estudo tem entre 30-38 anos, são brasileiras, naturais do estado do Rio de Janeiro, três possuem ensino fundamental incompleto e três, ensino médio incompleto, são casadas e referiram renda familiar média de dois salários mínimos. Apresentam a condição crônica de saúde relacionada à diabetes ou hipertensão, três são primíparas e outras três relataram ter realizado aborto em gestações anteriores experienciando complicações ao longo da gestação.
As 06 participantes relataram uso contínuo de medicação, não fazerem uso de álcool e cigarro e nem realizarem atividade física.

\section{Demandas de Acolhimento Emocional}

Observamos, ao iniciar o grupo, que as gestantes iam chegando para participar da nossa oficina com um olhar sério e inquieto, muitas estavam preocupadas com a consulta de pré-natal. Conforme os relatos eram expostos, misturavam-se a choros, desabafos e sensação de alívio. Percebemos, então, que o ambiente anterior, de possível tensão, havia se transformado em um espaço para troca de experiência e acolhimento emocional, o que não acontecia no ambulatório de pré-natal, segundo informações colhidas.

Nesse tema as gestantes evidenciaram um dos grandes desafios que encontraram no decorrer da gestação de alto risco: a falta de acolhimento emocional durante a assistência prestada no pré-natal. $\mathrm{O}$ acolhimento constitui-se em um desafio na construção de um cuidado integral, sendo o elemento de fundamental importância para a qualidade do serviço. É considerado ferramenta essencial para o estabelecimento de um processo de trabalho diferenciado e para a concretização de relações humanitárias entre usuários e profissionais (Araújo, Andrade, e Melo, 2011).

Assim, o acolhimento emocional está diretamente relacionado a uma escuta sensível, transpessoalidade, disponibilidade e empatia, dessa forma, os enfermeiros juntamente com o serviço de saúde devem contribuir para a resolutividade das demandas, valorizando as queixas e identificando as reais necessidades emocionais que atingem essas mulheres.

Abaixo segue a fala de algumas das gestantes:

Eu acho que elas deveriam parar pra escutar a gente, é uma maneira de desabafar, quando a gente desabafa consegue ficar mais leve. (Vida e Inspiração)

Eu acho que é um trabalho que deveriam fazer com a gente. Não é um exercício físico, é um exercício para a mente, para a gente relaxar e tentar sair daqui melhor. (Vida minha, Ezequiel)

Então eu acho que deveriam tirar um dia só para a gente vir aqui no ambulatório relaxar, conversar e desabafar. Acho que deveria ter isso aqui. (Filhote e Paixão)

$\mathrm{Eu}$ acho que seria muito legal se tivesse um acompanhamento emocional, um grupo pra gente sentar e conversar. (Mãe)

Eu acho que elas deveriam... Já que a gente está dizendo que está se sentindo ruim, que alguém trabalhasse isso com a gente, o emocional, a gente ficaria mais segura. (Filhote e Paixão) 


\section{DISCUSSÃO}

Embora tanto se discuta hoje sobre a humanização da assistência, onde devemos compreender o indivíduo de forma integral e não mais fragmentado, ainda sim, nos deparamos com um cuidado distanciado do que é preconizado, baseado nos preceitos de assistência do modelo biomédico, onde o foco do tratamento está centralizado na doença e como esta pode influenciar de forma negativa na vida da gestante/bebê, sendo negligenciados pelos enfermeiros os aspectos emocionais que envolvem essa mulher. Os enfermeiros focalizam seu tratamento nos aspectos fisiológicos das gestantes como avaliação de peso, mensuração da altura uterina, verificação da pressão arterial, ausculta dos batimentos fetais, orientação quanto à alimentação correta e a prática de exercícios físicos, não demonstrando interesse pelos aspectos emocionais destas gestantes (Oliveira, 2008).

De acordo com a Política Nacional de Humanização do SUS (Ministério da Saúde, 2006, p. 35), acolhimento é: "Recepção do usuário, desde sua chegada, responsabilizando-se integralmente por ele, ouvindo sua queixa, permitindo que ele expresse suas preocupações, angústias, e, ao mesmo tempo, colocando os limites necessários, garantindo atenção resolutiva e a articulação com os outros serviços de saúde para a continuidade da assistência, quando necessário." A atenção às gestantes é uma das propostas do acolhimento, sendo oferecido toda vez que a usuária procura o serviço de saúde e tem suas necessidades atendidas, seja por meio da assistência ou através de orientações, ou seja, quando a equipe multiprofissional demonstra interesse pelos seus problemas e se empenha na busca de soluções (Oliveira e Madeira, 2011). Azevedo, Araújo, Costa e Júnior (2009) em seu estudo observou que as gestantes classificadas como alto risco em atendimento ambulatorial se apresentavam visivelmente ansiosas com o desenrolar de sua enfermidade, e não encontravam espaço para conversar especialmente sobre questões ligadas à dimensão emocional da doença durante a consulta. Revelar como se dá a assistência às gestantes em todo o seu contexto não é algo tão simples. Esse obstáculo se deve aos poucos estudos referentes à comunicação entre as gestantes e médicos, enfermeiros e nutricionistas, assim como ao número limitado de locais onde a assistência à gestante seja desenvolvida por uma equipe multiprofissional (Oliveira e Madeira, 2011). O pré-natal de alto risco é realizado em blocos, separado por especialidade, onde em nenhum momento entre um atendimento e outro ou até mesmo no final de cada plantão é discutido com o enfermeiro o plano terapêutico dessa mulher e a melhor forma de atingir com êxito a integralidade da assistência.
Se o enfermeiro focasse o atendimento nas queixas relatadas pelas pacientes, além dos aspectos fisiológicos da doença, esquecendo um pouco a anamnese tradicional, provavelmente as gestantes sairiam mais tranquilas, satisfeitas e preparadas para lidar com qualquer tipo de adversidade no decorrer da gestação (Azevedo et al., 2009).

\section{CONCLUSÃO}

Este estudo debruçou-se essencialmente sobre o aspecto emocional das gestantes que convivem com algum tipo de doença crônica, buscando encontrar estratégias de promoção à saúde mental destas mulheres.

Os depoimentos revelaram a necessidade de escuta ativa aos anseios em todo o seguimento da gestação de alto risco, onde as mulheres se encontram fragilizadas e instáveis, e diante de um atendimento tão robotizado e fragmentado não encontram espaço para exporem seus medos, angústias e expectativas do que é ser gestante e conviver com uma doença crônica. Percebemos que é através da interação entre o enfermeiro e a gestante durante a rotina de consultas do pré-natal que podemos nos apropriar das reais necessidades emocionais destas mulheres. Certificamo-nos, assim, que se torna cada vez mais essencial uma assistência fundamentada na humanização, nas políticas nacionais de saúde e que traz a integralidade como ponto chave da qualidade da assistência prestada. Contudo, diante dos aspectos e experiências aqui relatadas, percebemos a necessidade de transformação da conduta do serviço de saúde no processo de busca por uma assistência de qualidade no que tange a abordagem do estado emocional e os aspectos que envolvem a subjetividade dessa mulher. Para tal, se fazem fundamentais a escuta sensível e o uso da visão holística.

\section{IMPLICAÇÕES PARA A PRÁTICA CLÍNICA}

A condição clínica pré-existente a gestação pode desencadear uma série de questionamentos envolvendo o futuro de sua gravidez interligado a sentimentos como ansiedade, medo, insegurança e impotência, que resultam em um estado de desequilíbrio emocional. Tendo em vista a instabilidade psíquica, se torna essencial uma assistência de enfermagem de qualidade a fim de propiciar um meio em que as mesmas se sintam acolhidas, escutadas, compreendidas, e que somado a um cuidado humanizado possam ter o suporte emocional necessário para enfrentar os possíveis obstáculos da gestação. 


\section{REFERÊNCIAS BIBLIOGRÁFICAS}

Araújo, M. A. L., Andrade, R. F. V., e Melo, S. P. (2011). $\mathrm{O}$ acolhimento como estratégia de atenção qualificada: Percepção de gestantes com hiv/aids em Fortaleza. Revista Baiana de Saúde Pública, 35(3), 710-721.

Arrais, A. R., Mourão, M. A., e Fragalle, B. (2014). O pré-natal psicológico como programa de prevenção à depressão pós-parto. Saúde e Sociedade, 23(1). Disponível em http://www.scielo.br/pdf/sausoc/ v23n1/0104-1290-sausoc-23-01-00251.pdf

Azevedo, D. V., Araújo, A. C. P. F., Costa, I. C. C., e Júnior, A. M. (2009). Percepções e sentimentos de gestantes e puérperas sobre a pré-eclâmpsia. Revista de Salud Pública, 11, 347-358. Disponível em http://www. scielosp.org/pdf/rsap/v11n3/v11n3a04.pdf

Bardin, L. (2009). Análise de conteúdo (5 $5^{\mathrm{a}}$ ed.). Lisboa: Edições 70.

Caldas, D. B., Silva, A. L. R., Böing, E., Crepaldi, M. A., e Custódio, Z. A. O. (2013). Atendimento psicológico no pré-natal de alto-risco: A construção de um serviço. Psicologia Hospitalar, 11(1). Disponível em http://pepsic.bvsalud.org/pdf/ph/v11n1/v11n1a05.pdf

Duarte, S. J. H. (2012). Motivos que levam as gestantes a fazerem o pré-natal: Um estudo das representações sociais. Ciencia y EnfermerIa XVIII, 18(2). Disponível em http://www.scielo.cl/pdf/cienf/v18n2/art_08.pdf

Guerra, M., Braga, M., Qulhas, I., e Silva, R. (2014). Promoção da saúde mental na gravidez e no pós-parto. Revista Portuguesa de Enfermagem de Saúde Mental (Esp. 1).

Lima, Y. M. S., e Moura, M. A. V. (2005). Consulta de enfermagem pré-natal: A qualidade centrada na satisfação da cliente. Revista Cuidado é Fundamental, 9(1/2), 9399. Disponível em https://www.researchgate.net/publication/267950271_Consulta_de_Enfermagem_prenatal_a_qualidade_centrada_na_satisfacao_da_cliente

Maçola, L., Vale, I. N., e Carmona, E.V. (2010). Avaliação da autoestima de gestantes com uso da Escala de Autoestima de Rosenberg. Revista Escola Enfermagem, 44(3). Disponível em http://www.scielo.br/pdf/reeusp/ v44n3/04.pdf

Ministério da Saúde. Secretaria de Atenção à Saúde. Núcleo Técnico da Política Nacional de Humanização. (2006). HumanizaSUS: Documento base para gestores e trabalhadores do SUS ( $3^{a}$ ed.). Brasília: Editora do Ministério da Saúde. Disponível em http://bvsms.saude. gov.br/bvs/publicacoes/documento_base.pdf
Ministério da Saúde. Secretaria de Atenção à Saúde. Departamento de Ações Programáticas Estratégicas. (2012). Gestação de alto risco: Manual técnico ( $5^{\mathrm{a}} \mathrm{ed}$.). Brasília: Editora do Ministério da Saúde.

Moraes, L. M. P., Braga, V. A. B., e Silveira, L. C. (2003). Sociopoética e enfermagem: Uma aproximação na pesquisa com adolescentes. Revista Baiana de Enfermagem, 18(1). Disponível em http://www.portalseer.ufba. br/index.php/enfermagem/article/view/3875

Oliveira, V. J. (2008) Vivenciando a gravidez de altorisco: Entre a luz e a escuridão (Dissertação de Mestrado em Enfermagem). Belo Horizonte: Escola de Enfermagem da Universidade Federal de Minas Gerais. Disponível em https://www.nescon.medicina.ufmg.br/ biblioteca/imagem/2047.pdf

Oliveira, V. J., e Madeira, A. M. F. M. (2011). Interagindo com a equipe multiprofissional: As interfaces da assistência na gestação de alto risco. Escola Anna Nery, 15(17). Disponível em http://www.scielo.br/pdf/ean/ v15n1/15.pdf

Quevedo, M. P., Lopes, C. M. C., e Lefèvre, F. (2006). Os significados da maternidade para mulheres cardiopatas e diabéticas com gravidez de risco. Revista Brasileira Crescimento e Desenvolvimento Humano, 16(1). Disponível em http://pepsic.bvsalud.org/pdf/rbcdh/ v16n1/03.pdf

Quevedo, M. P. (2010). Experiências, percepções e significados da maternidade para mulheres com gestação de alto risco (Tese de Doutorado em Saúde Pública). São Paulo: Universidade de São Paulo. Disponível em http://www.teses.usp.br/teses/disponiveis/6/6135/tde14052010-082745/pt-br.php

Santos, E. V. (2014). A importância do pré-natal e o papel do enfermeiro neste contexto. Disponível em http://www.portaleducacao.com.br/enfermagem/ artigos/57913/a-importancia-do-pre-natal-e-o-papeldo-enfermeiro-neste-contexto

Shimizu, H. E., e Lima, M. G. (2009). As dimensões do cuidado pré-natal na consulta de enfermagem. Revista Brasileira de Enfermagem, 62(3), 387-392. doi: dx.doi. org/10.1590/S0034-71672009000300009

Szenészi, G. V. (2011). Está na hora?: A psicologia das linhas do tempo: Aplicações à vida pessoal, à terapia e ao coaching. Florianópolis, Santa Catarina: Editora Metaprocessos.

Vasques, F. A. P. (2006). Pré-natal: Um enfoque multiprofissional. Rio de Janeiro: Rubio Editora. 